\title{
Measuring the Resonance Structure of Accelerator Impedance with Single Bunches
}

\author{
T. Bohl, T. P. R. Linnecar, and E. Shaposhnikova \\ CERN/SL, CH-1211 Geneva 23, Switzerland
}

(Received 15 August 1996)

\begin{abstract}
We describe a new method used in the CERN SPS accelerator to measure the longitudinal impedance in the frequency range $100 \mathrm{MHz}$ to $4 \mathrm{GHz}$. Single high intensity proton bunches were injected and their spectrum observed during slow debunching. The presence of different resonant impedances leads to line density modulation at the resonant frequencies. This instability reaches some maximum modulation amplitude which was recorded as a function of frequency for many bunches. Using sufficiently long bunches the SPS impedance structure was observed and previously unknown sources were identified. [S0031-9007(97)02916-5]
\end{abstract}

PACS numbers: 29.27.Bd

Beam intensity in particle accelerators is usually limited by collective instabilities which are the result of electromagnetic interaction of the beam with its environment. This interaction can be described by a coupling impedance $Z(\omega)$, the Fourier transform of the wake potential, proportional to the electric field integrated over one turn as seen by a probe particle following a source particle at time interval $t$. Knowledge of the impedance allows the behavior of intense beams to be explained or predicted $[1,2]$.

The main intensity limitation for the proton beam in the SPS, future injector for the Large Hadron Collider (LHC), comes from a longitudinal instability of a single bunch, known as the "microwave instability" since it is associated with the growth of high frequency signals. In this Letter we present results of measurements from which the longitudinal coupling impedance over a wide frequency range has been determined, and the source of the instability identified. Below we consider only longitudinal beam motion (and impedance), which in this case can be treated separately from transverse motion.

Many of the vacuum chamber elements through which the beam travels have a cavitylike structure whose impedance can be represented as the sum of several individual resonances of the form

$$
Z(\omega)=\frac{R_{\mathrm{sh}}}{1+i Q\left(\omega / \omega_{r}-\omega_{r} / \omega\right)},
$$

characterized by a resonant frequency $\omega_{r}=2 \pi f_{r}$, shunt impedance $R_{\mathrm{sh}}$, and quality factor $Q$.

Measurements of impedance with a single bunch can give information only about the effective impedancethe actual impedance integrated over the spectrum of the bunch.

For a stable bunch the effective impedance is defined by integration over the initial bunch spectrum centered at zero frequency. This effective impedance can be estimated from bunch lengthening (shortening) measurements based on the potential well distortion effect. Since the width of the bunch spectrum is inversely proportional to the bunch length, long bunches "see" only the low frequency part of the coupling impedance. Difficulties in resolving the "fine structure" of the machine impedance led to the creation of the widely used broadband impedance model, which replaces this structure by one resonator with very low $Q$ (usually 1 ) and resonant frequency defined by the beam pipe cutoff frequency. The parameters of the SPS broadband model deduced from this type of measurement are $Q=1, R_{\mathrm{sh}}=0.3 \mathrm{M} \Omega$, and $f_{r}=1.3 \mathrm{GHz}$. This model has been used up to now also to describe the microwave instability.

For an unstable bunch the situation is different. The growth rate of some mode $m$ depends on the effective impedance which is now defined by integration over the spectrum of this mode with a nonzero center frequency. In this way the growth rate of the head-tail instability was used to estimate the effective transverse impedance [3].

Our method is based on the measurement of the spectrum of unstable modes. Interaction of the intense bunch with different resonant impedances in the machine leads to instability resulting in bunch line density modulation at the resonant frequencies. The instability reaches some maximum modulation amplitude which was recorded during the measurements. The mode spectrum has a center frequency close to the resonant frequency of the impedance and width given both by bunch length and impedance width $\left[\omega_{r} /(2 Q)\right]$. Hence long bunches allow better resolution of resonant peaks.

Above the threshold intensity, instability starts in the regime where the bunch can be treated as monoenergetic. Later with the increase in energy spread the instability saturates. Nonlinear saturation of the unbunched coasting beam instability has been analyzed in [4]. We used numerical simulation to model the nonlinear stage of this process for a bunched beam.

To describe the initial phase of the instability, a linear theory can be applied. In our experiment bunches were injected into the accelerator with no external radiofrequency voltage ( $\mathrm{rf}$ off) and started to spread out or debunch. If we assume that debunching is sufficiently slow, then the distribution function in longitudinal phase space can be divided into an unperturbed, time independent, part $F(u, \theta)$ and a perturbed part $f(u, \theta, t)$. Here $\theta$ is the azimuthal angular coordinate around the machine 
and $u=\Delta E$ the energy deviation relative to the reference particle at the bunch center having angular revolution frequency $\omega_{0}$ and energy $E_{0}$.

The equations of particle motion are

$$
\begin{gathered}
\dot{\theta}=k_{0} u, \\
\dot{u}=-\frac{\left(e \omega_{0}\right)^{2}}{2 \pi} \sum_{n}\left(\rho_{n}+G_{n}\right) Z_{n} \exp (\operatorname{in} \theta),
\end{gathered}
$$

where $k_{0}=\eta \omega_{0} / E_{0}, \eta=1 / \gamma_{t}^{2}-1 / \gamma^{2}$, and $\gamma_{t}$ is the Lorentz factor at transition energy. The right side of Eq. (3) contains the voltage induced by the beam which depends on the coupling impedance $Z_{n}(\omega)=Z\left(n \omega_{0}+\right.$ $\Omega$ ) and on the Fourier transform of the bunch line density, with unperturbed part $G_{n}$, and perturbed part

$$
\rho_{n}=\frac{1}{2 \pi} \exp (-i \Omega t) \iint f(u, \theta) \exp (-i n \theta) d \theta d u,
$$

where the growth rate of the instability is given by the imaginary part of the coherent oscillation frequency $\Omega$.

Using the linearized Vlasov equation leads to the same matrix equation as in the analysis of a fast microwave instability when, in a system with rf on, the synchrotron motion can in fact be neglected [5]. For a monoenergetic bunch this equation can be written in the form

$$
\rho_{m}=-i \frac{k_{0} m}{2 \pi}\left(\frac{e \omega_{0}}{\Omega}\right)^{2} \sum_{n} G_{m-n} Z_{n} \rho_{n} .
$$

We consider a resonant impedance [see Eq. (1)] centered at harmonic $n_{0}=\omega_{r} / \omega_{0}$ with $\omega_{r} \tau \gg 1$, where $\tau$ is the bunch length in time. In this case we can ignore the voltage induced by the unperturbed bunch spectrum. If the impedance bandwidth is much less than the bunch spectrum width $\propto 1 / \tau$, so that $1 \ll \omega_{r} \tau \ll 2 Q$, then the bunch spectrum in Eq. (5) can be assumed constant over the impedance width with $G_{m-n} \simeq G_{m-n_{0}}$. In this approximation the growth rate can be obtained by summation over $m$ in Eq. (5):

$$
\operatorname{Im} \Omega \simeq \omega_{r}\left(\frac{N e^{2} \omega_{0}|\eta|}{16 \pi E_{0}} \frac{R_{\mathrm{sh}}}{Q}\right)^{\frac{1}{2}},
$$

where $N$ is the number of particles in the bunch. This growth rate is defined by the resistive (real) part of the impedance. The contribution coming from the imaginary part is $\omega_{r} \tau / Q$ times smaller. If $\operatorname{Im} \Omega / \omega_{r} \gg|\eta| \Delta E / E_{0}$, the assumption of slow debunching is justified.

From Eq. (5), the spectrum of the unstable mode for a bunch with Gaussian line density is

$$
\rho_{n} \sim n G_{n-n_{0}} \sim n \exp \left[-\frac{\sigma^{2}\left(n-n_{0}\right)^{2}}{2}\right],
$$

with width $\propto 1 / \sigma$ and a maximum shifted from $n_{0}$ by $1 / n_{0} \sigma^{2}$. Here standard deviation $\sigma \simeq \omega_{0} \tau / 4$.

As one can see from Eq. (7) it is possible to determine the resonant frequencies of the impedances from the spectrum of the unstable bunch. The growth rate, Eq. (6), can give $R_{\mathrm{sh}} / Q$ for a known resonant frequency $\omega_{r}$. However, due to the complicated structure of the signal

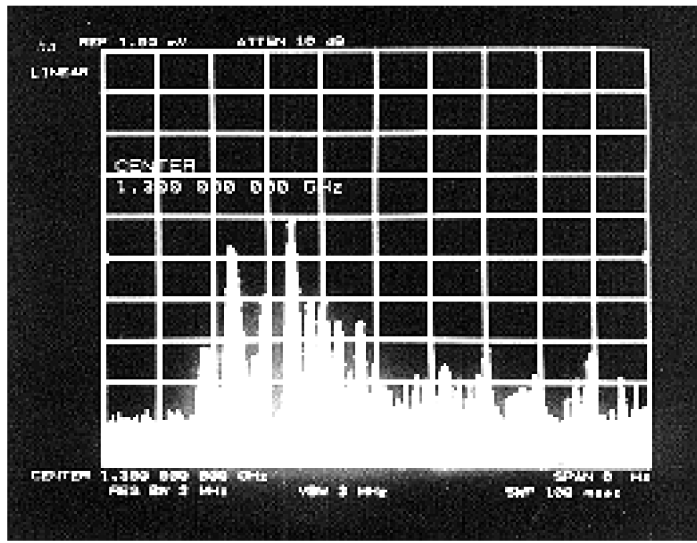

FIG. 1. Bunch spectrum component at $1.3 \mathrm{GHz}$ as a function of time, $10 \mathrm{~ms} / \mathrm{div}$, for a bunch with $\tau=4 \mathrm{~ns}$ and $N=$ $3.5 \times 10^{9}$. Injection at $12 \mathrm{~ms}$.

in time, varying from shot to shot (see a typical example in Fig. 1), the growth rate is, in general, ill defined in comparison to the maximum value of the mode amplitude.

In our measurements the spectrum of unstable modes was recorded for a few sets of bunch parameters. In Fig. 2 we present some data obtained with relatively short (upper curve) and long (lower curves) bunches.

Short bunches had longitudinal emittance $\varepsilon=0.03 \mathrm{eVs}$, length $\tau=4 \mathrm{~ns}$, and average intensity $N=3.5 \times 10^{9}$. Long bunches had $N=1.2 \times 10^{10}$ with $\varepsilon=0.35 \mathrm{eVs}, \quad \tau=28 \mathrm{~ns}$ (dashed line), and $\varepsilon=0.24 \mathrm{eV} \mathrm{s}, \tau=25 \mathrm{~ns}$ (solid line). The beam energy was $26 \mathrm{GeV}$, above transition energy $\left(\gamma_{t}=23.4\right)$. The signal from a wall current pickup (bandwidth $4 \mathrm{GHz}$ ) was scanned from $100 \mathrm{MHz}$ to $2 \mathrm{GHz}$, with an average step of $50 \mathrm{MHz}$, using a spectrum analyzer of bandwidth $3 \mathrm{MHz}$. At each frequency the maximum amplitude

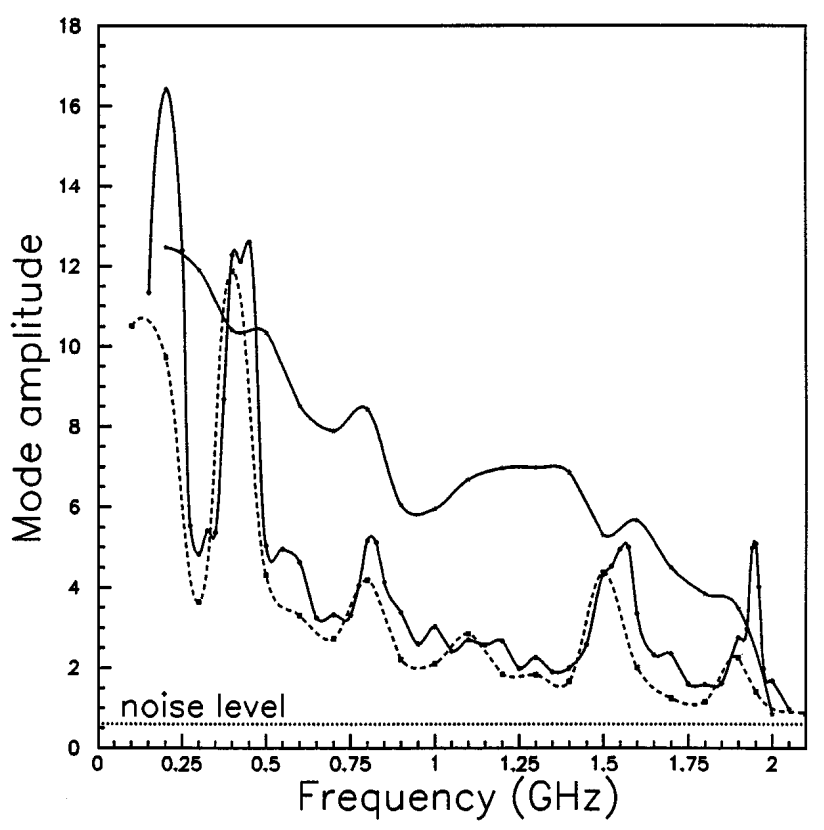

FIG. 2. Spectral distribution measured with short (upper curve) and long (lower curves) bunches. 
of the signal within the first $100 \mathrm{~ms}$ after injection was recorded. The data were taken for at least ten different bunches and then averaged. For the long bunches with maximum $\Delta E / E_{0} \simeq \pm 2 \times 10^{-4}$ the increase in bunch length during the observation time did not exceed $50 \%$.

As expected, see Eq. (7), measurements with short bunches result in a maximum amplitude spectrum of

(a)

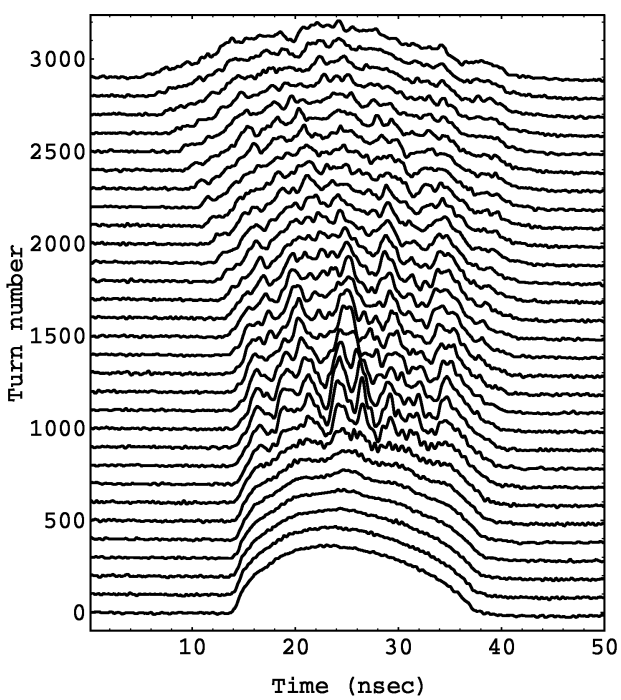

(b)

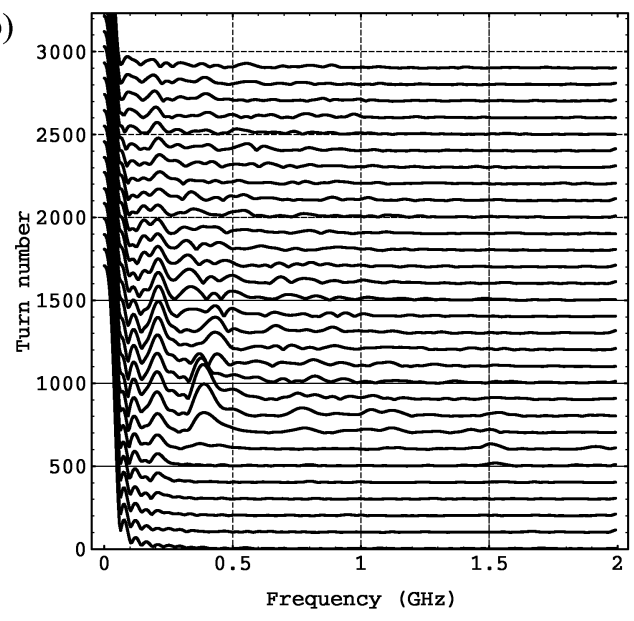

(c)

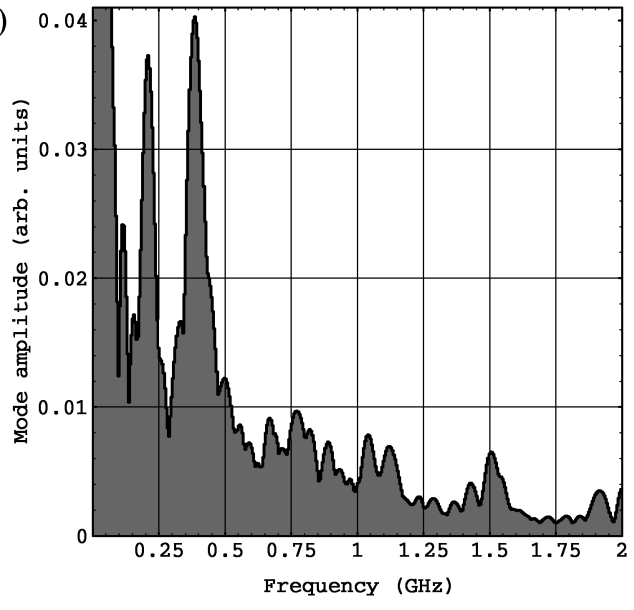

FIG. 3. Fourier analysis of longitudinal bunch profiles. relatively low resolution compared to the data with long bunches. Additional smearing of the peaks comes from the faster debunching of the short bunches. Nevertheless both spectra show common features. Measurements with the different long bunches in Fig. 2 are very similar.

As another approach, we also acquired the longitudinal bunch profile (scope sampling rate $4 \mathrm{GHz}$ ) at regular time intervals. The profiles in Fig. 3(a) were taken every 100 turns $(2.3 \mathrm{~ms})$. The growth of the instability can clearly be seen as well as the slow debunching. Each of these profiles was Fourier analyzed. In this way the growth of the unstable modes with time in the frequency domain is also seen; see Fig. 3(b). In Fig. 3(c) the projection of the Fourier spectra of all bunch profiles from Fig. 3(b) is shown. The mode amplitude reaches its maximum before debunching is significant. Using data from many bunches in this way should give the same results as with the first method but with even better resolution. In this particular example we already see a rich mode structure.

The same method used to analyze the experimental data was applied to data obtained by simulation with the code ESME [6], which tracks turn by turn the bunch motion in the accelerator using macroparticles. Some results of simulations with a single resonator at $f_{r}=$ $1.6 \mathrm{GHz}$ in the SPS are shown in Fig. 4. The resistive part of the impedance when $Q=100$ is shown in arbitrary units as a dashed line. From these examples we can see that the mode amplitudes peak at a slightly higher frequency than $f_{r}$. This shift also increases with intensity. The peak amplitude grows in a nonlinear way with increasing $R_{\mathrm{sh}} / Q$ and bunch intensity. With increasing intensity the amplitude at the second harmonic

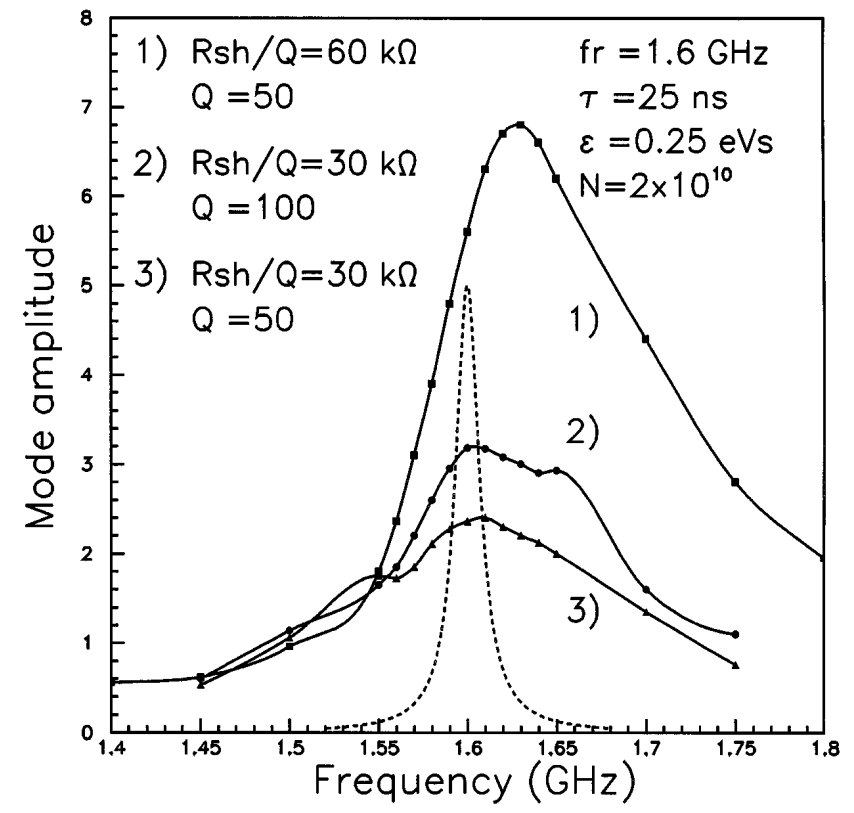

FIG. 4. Spectral distribution obtained from simulations for resonance impedance with different parameters. 


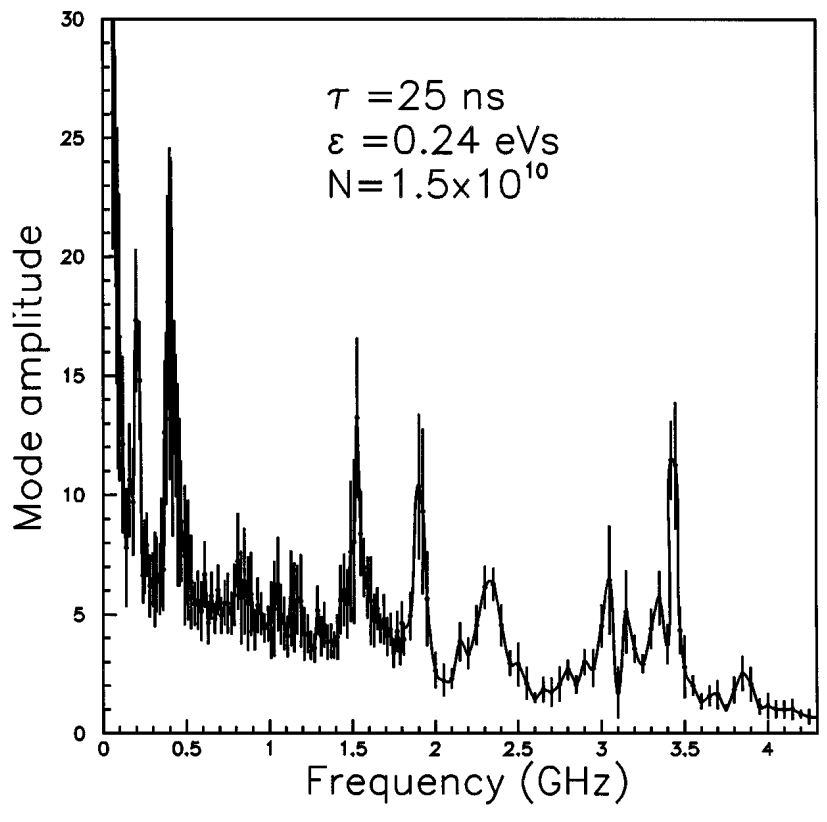

FIG. 5. Measured spectral distribution.

of the resonator frequency also grows. Therefore care should be taken when interpreting the experimental data.

We used the method described above to analyze the fine structure of the SPS impedance over a wide frequency range. The results are presented in Fig. 5. The peaks observed were identified later with different sources of impedance in the accelerator. Their resistive parts, calculated from hardware, are shown in Fig. 6.

In the frequency range from $100 \mathrm{MHz}$ to $1.2 \mathrm{GHz}$ impedance sources are the fundamental and higher order modes of the six different rf systems installed in the SPS. They have been found by calculations and laboratory measurements and presented in many references which

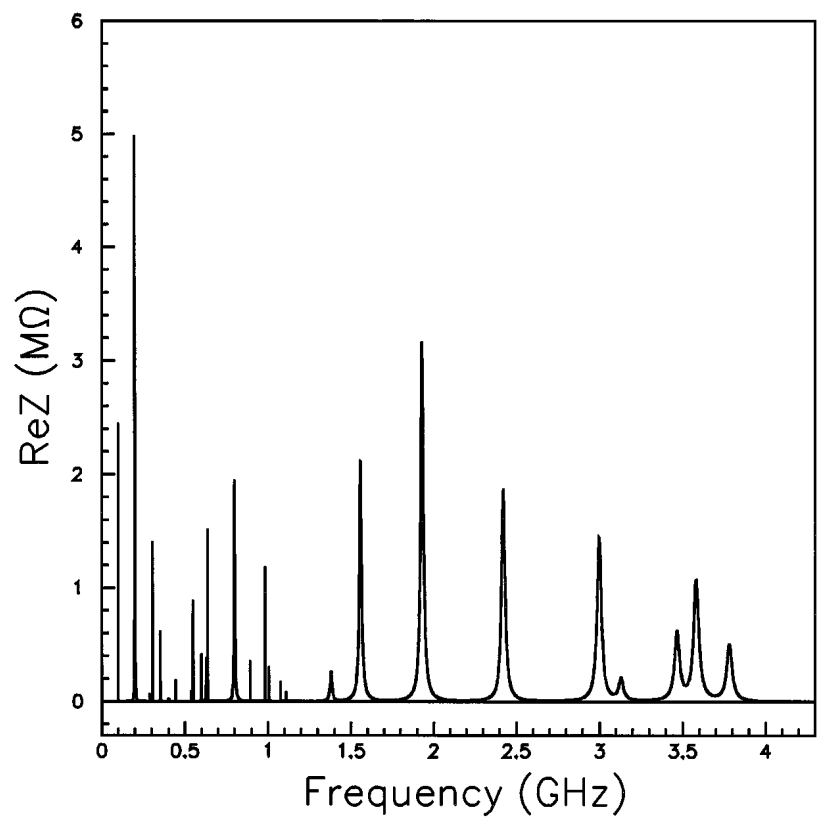

FIG. 6. Calculated resonant impedances. are summarized in [7]. Most of these impedances are damped to low $Q$ values so that they do not cause coupled bunch mode instabilities but, having high $R_{\mathrm{sh}} / Q$, can still be seen by a single bunch. Note that in Fig. 6 the impedance corresponding to the large peak at $400 \mathrm{MHz}$ in Fig. 5 is missing. This source was identified after our measurements and can be attributed to the extraction septa. Laboratory measurements on a spare septum, as well as numerical calculations, show the same frequency structure situated around $400 \mathrm{MHz}$, explaining well the complex structure seen in measurements with the beam in Figs. 3(c) and 5.

The main source of impedance from 1.4 up to $4 \mathrm{GHz}$ are the vacuum ports which have a cavitylike structure due to their connection to smaller diameter vacuum pipes on either side. In the ring there are about 800 devices of a similar type and 100 which are shorter or longer. In Fig. 6 the resistive part of the impedance calculated for the main type of vacuum port is presented. Every peak found by the numerical code can also be seen in measurements with the beam. Because of the small vertical dimensions of the connecting beam pipes, modes up to $4 \mathrm{GHz}$, cutoff frequency of these pipes, are still trapped in these accidental cavities [8]. The vacuum ports are considered now to be the main cause of the microwave instability of intense single bunches in the SPS.

These results obtained for the SPS allow the existing broadband impedance model to be replaced by a more realistic model using the dominant impedances found. The results indicate where changes must be made in the accelerator to reduce these impedances, of great importance in preparing the SPS as LHC injector. We also believe that the techniques described here can be used in other accelerators to observe the detailed structure of the machine impedance.

We are grateful to the CERN PS operations group for the various beams and to those who helped to identify the sources of different impedances. We also thank D. Boussard for his useful comments.

[1] A. Chao, Physics of Collective Beam Instabilities in High Energy Accelerators (J. Wiley \& Sons, New York, 1993).

[2] A. Hofmann, in Proceedings of the Vth European Particle Accelerator Conference, Sitges, 1996 (Institute of Physics, Bristol, 1996), p. 143.

[3] J. Gareyte and F. Sacherer, in Proceedings of the IXth International Conference on High Energy Accelerators, Stanford, 1974 (National Technical Information Service, Springfield, VA, 1974), p. 341.

[4] Y. Chin and K. Yokoya, Phys. Rev. D 28, 2141 (1983).

[5] J. M. Wang and C. Pellegrini, in Proceedings of the XIth International Conference on High Energy Accelerators, Geneva, 1980 (Birkhauser-Verlag, Basel, 1980), p. 554.

[6] J. MacLachlan, Fermilab Report No. TM-1716, 1991.

[7] T.P. R. Linnecar and E. Shaposhnikova, CERN Report No. SL/Note 96-46(RF), Geneva, 1996.

[8] W. Höfle, CERN Report No. SL/Note 96-40 (RF), Geneva, 1996. 